\title{
Cognitive Analysis of the "Discourse Stances" in English News Reports on Smog in China and America
}

\author{
Wenhui Yang ${ }^{1}$, Linyan Cheng ${ }^{1} \&$ Kaiyue Zhen ${ }^{2}$ \\ ${ }^{1}$ International College, Guangdong University of Foreign Studies, Baiyun Dadao North No. 2. Guangzhou, \\ Guangdong, 510420, China \\ ${ }^{2}$ Faculty of Business and Economics, University of Melbourne, 111 Barry St., Carlton VIC 3053, Melbourne, \\ Australia \\ Correspondence: Wenhui Yang, International College, Guangdong University of Foreign Studies, Guangzhou, \\ Guangdong, 510420, China. E-mail: 198910321@oamail.gdufs.edu.cn
}

Received: April 20, 2020

Accepted: May 21, 2020

Online Published: May 27, 2020

doi:10.5539/ijel.v10n4p145

URL: https://doi.org/10.5539/ijel.v10n4p145

\begin{abstract}
This analysis contrasts on Chinese smog news (CSN) with American smog news (ASN), probing into the complicated discourse stances and their represented cognitive mechanism. Having been assisted by "glossary extraction", the authors uncover the correlation between varied stance glossaries and the hidden cognitive mechanisms. The research provides hints on social cognition in news encryption and decryption, based on the database of thirty pieces of news reports from Chinese news agencies and thirty from American sources respectively. The analytical results reveal that Chinese news frequently quotes the comments of officials and is largely dominated by official and political stances of government, whilst American news frequently features occupational and public stances with pervasive individual and personal tones, attitudes, and dictations. This cognitive research on English weather news reports casts light on the discrepancies and commonalities in the adoption of stance glossaries in media discourse, drawing respective cognition construction of media writers from different cultures, which further illustrates how public cognition being framed on social issues in discourses.
\end{abstract}

Keywords: ASN, cognitive pattern, CSN, discourse stance, stance glossary

\section{Research Background}

Since 2012, the prevalence of choking air in metropolitan cities like Beijing and Shanghai has given rise to heated daily discussions among the general public. On the social media portal website Micro-Blog, the Chinese equivalent of "Twitter", the once unfamiliar word "smog" to the Chinese has turned into a trendy topic, being posted by numerous bloggers with hash-tags. Given that inhabitants in Beijing, Shanghai, and other large populous cities have been plagued by "smog" and "disturbing air" from time to time, smog has gradually become a core topic in daily talks, and is often reported on the front pages of newspapers both at home and abroad, sweeping the whole nation and becoming one of the major concerns in China during recent years. Although a few studies have centered on climate issues from social and economic perspectives, the analyses from the perspective of cross-cultural linguistics are rather rare, which shed light on the necessity of this research, drawing from the perspectives of cognitive stance and frame. As Biber et al. (1999) point out, stance is an interaction between the writers and the contents, as well as the audience, while "frame represents the knowledge and beliefs pertaining to specific and frequent recurring situations" (Ungerer \& Schmid, 2001). Both "stance" and "frame" reveal a pragmatic relation between linguistic elements context and personal thinking patterns, feelings, attitudes, value judgments, and assessments of writers or speakers, and such views are also supported by Kärkkäinen (2012). News is normally perceived as reporting facts and should be as objective as possible. However, the reality is that the way news is structured and reported is susceptible to social backgrounds, as news reports are not "objective", even in weather reports (Vannini \& Mccright, 2007). Being a relatively new area in linguistics, cognitive discourse stance and frame studies have not yet engulfed the field of discourse analysis, leaving much space for further in-depth research. In recent years, frictions have often arisen between China and America, with the former criticizing the latter for their prejudices and double-standards in reporting Chinese affairs. To differentiate from other traditional studies, the present study will, from a perspective of cognitive 
discourse and with a glossary extraction approach, analyze the following questions: What are the lexical stance glossaries or keywords adopted in Chinese smog news (CSN) and American smog news (ASN) reports respectively? How do they reflect various discourse stances in smog news? And how are the stance differences associated with the reporters' different cognitive construction in news reports on smog?

\section{Literature Review and Theoretical Framework}

\subsection{Cognitive Studies of News Discourse}

The notion of cognition has been widely employed in various fields over the years, including psychology, artificial intelligence, sociology, and applied linguistics. Serving as a psychological device, the cognitive study offers insights into the psychological activities and it manipulates an exerting influence on subsequent judgment (Rhoads, 2004). Among scholars, Bell (1991, pp. 36-44) argues that the lexicon plays a key role in language applications, describing the many-handed and multilayered nature of news production, which requires an expansion of the number of roles of the speaker: to principal, author, editor, and animator. In contrast, Fowler (1991, p. 139) observes that dominant and codominant, competing, officially sanctioned ideologies are the stuff of the socio-cognitive fabric of media language. To rend this fabric might require a "major, bold, and self-conscious shift of discourse". This shift seems to be more convincing, on account of current research findings on cognitive discourses analysis, that news media discourses certainly reflect news writer's cognition of the world, and affect news readers' cognitive construction about the world. Bednarek and Caple (2017) persuasively illustrate how news events are retold in the news discourses and made 'newsworthy' through both semiotic resources of language and image, bringing together corpus linguistics and multimodal discourse analysis in a stimulating and unique book for researchers in linguistics, semiotics, critical discourse analysis and media/journalism studies. Cognition is commonly utilized to effect persuasion in news discourse by news composers. Political and economic news discourses are often the foci in related studies. The research into the political disputes of the 2008 American presidential campaign conducted by Entman (2010) proves that the interaction of bodily experience with the world, cultural norms, and journalistic ethnics gives rise to a slanted lexis.

Similarly, Lukin (2006), by analyzing corpus news discourse, argues that 'bias' and its related linguistic lexical items are more likely to remain a political weapon than a tool for understanding how the media shapes people's experience of crucial events. She claims that "the only dimension of 'bias' on which all who use it would agree is that it implies the notion of linguistic choice. In other words, when a charge of bias is made, the implication is that a process of selection - of particular words or phrases, of particular facets of a story over others, or regarding the reliance on particular kinds of sources to the background, or exclusion of others - is involved, and that other choices were available, but not taken up" (Lukin, 2006, p. 67). The biased choices on slanted lexis or salient information also show up in economic and sport news discourse to decipher the covert cognition embedded in the overt lexical usages, as argued by Yang et al. (2016, 2018a, 2018b). In a study to interpret China and its economy in American reports, Yang et al. (2016) probe into the use of cognitive force dynamics in news with an attempt to reveal the complicated correlation between language selections and cognition by means of paying closer examination to the linguistic stimuli, i.e. the lexical choices, which contribute to the initiation of the fixed frames and restored cognition based on the employment of positive/negative stimuli. Drawing insights on the interrelationship between lexical choices and cognition patterns from the empirical study carried out by Yang et al. (2016, 2018a, 2018b), the authors further explore the cognitive variations and language differences denoted in slanted lexis employed in English news discourse on "smog" in China from two different cultural backgrounds. These differences are analyzed with the hope of drawing a preliminary picture of varied social cognition and linguistic differences existing in both Chinese and American news discourses.

\subsection{Discourse Stance}

Discourse stance is a compulsory element to frame one's cognition, which mainly focuses on three levels: lexical, syntactic, and discoursal. From the lexical standpoint, Keck and Biber (2004) claim that modals and semi-modals are the most typical tools employed by speakers and writers in demonstrating their stance in spoken and written texts. At the syntactic level, Biber and Conrad (2001) apply corpus-based methods to study the ways in which speakers and writers use adverbials to mark their personal stances and define three major domains: epistemic stance which comments on the certainty, reliability or limitations of a proposition, attitudinal stance which comments on affect and evaluations, and style stance. Chandrasegaran and Kong (2007) discover the extent to which a group of high school students from a non-native English-speaking background are capable of engaging in stance assertion and stance-support behaviors in an argument on an online forum. The study reveals a considerable number of stance-support strategies ranging from assertion of personal belief to rebuttal of 
anticipated opposing views. Baratta (2009) proposes that passive stance is a rhetorical reality and, from the analysis of the students' written work, asserting that passive voice can be a subtle way for writers to express themselves in their essays. From a study of threatening acts, Gales (2011) identifies interpersonal stance in threatening discourse and arrives at a conclusion that people who threaten use multiple rhetorical strategies to convey interpersonal meaning and take stances that both strengthen and weaken their apparent level of commitment, thus contradicting the one-sidedness of threatening language ideologies, and demonstrating the need for further research on stance taking in threatening discourse.

From a discourse perspective, Hunston and Thompson (2000) raise the term "evaluation" as an up-and-coming field of study in which semantics, pragmatics, grammar and discourse overlap. They propose nine contributions to the volume under review and find a considerable range of lexical, grammatical and textual approaches to the expression of opinion and stance in English. These contributions include corpus-based studies of lexical and grammatical items carrying an evaluative load, evaluation in and of narrative, covert stance in persuasive rhetoric, a systemic-functional modeling of appraisal, and a layered discourse model to persuasive texts. Although Hyland (2005) holds that stance is composed of four main elements which are supported by stance glossary identification: hedges (e.g., possible), boosters (e.g., certainly), attitude (e.g., important) and self-mentions (e.g., I), his approach suffers in its insufficiency to provide information about where these features are likely to cluster. Nevertheless, studies related to stance are conducted from various perspectives, though stance realization and use of stance glossaries should be explored in a particular genre or text. Surprisingly, stance of news is rarely explored in news discourse studies. Central to the concept of stance is the point of view or position that a writer takes on an issue. The writer's position is a claim asserted "for general acceptance", and therefore "the starting point" of the argument. According to Chandrasegaran and Kong (2007), arriving at a stance or position requires the writer to interpret phenomena and exercise some form of evaluative judgment on the basis of desirability, benefit, or other criteria.

Based on the study of Biber et al. (1999), Berman (2005) fixates his attention on one dimension within stance: attitude, including epistemic attitude, deontic attitude or affective attitude.

Epistemic attitude: the writer's belief about the truth of a state of affairs. (For example, "Clearly, we have achieved our goals." / "I think that this is the least likely choice.")

Deontic attitude: an evaluative viewpoint. (For example, "The research must be very beneficial.")

Affective attitude: writer's emotions toward a state of affairs. (For example, "The film was tedious.")

An epistemic attitude concerns a relation between a cognizant speaker/writer and a proposition in terms of possibility, certainty, or the evidence for the individual's belief that a given state of affairs is true (or false), which is directly docked with epistemic stance proposed by Biber et al. (1999). A deontic attitude adopts a judgmental prescriptive or evaluative viewpoint in relation to the topic, which is often associated with modal words. An affective attitude, in contrast to the epistemic, concerns a relation between cognizant speakers/writers and their emotions (desire, anger, grief, etc.) with respect to a given state of affairs. Nevertheless, it should be noted that modal words are not limited to deontic attitude, owing to their complex uses and functions. On account of Biber et al.'s (1999) categorization and Berman's (2005) dimension within stance, the authors make a list of modal verbs and their stance attitudes in Table 1.

Table 1. Modals and their stances

\begin{tabular}{lll}
\hline Modal & Usages & Stances \\
\hline Can & ability, suggestion, request & evaluation/deontic \\
Be able to & ability & evaluation/deontic \\
May & possibility, formal request /permission & hedging/epistemic \\
Might & possibility & hedging/epistemic \\
Must & obligation, certainty that something is true & judgment/deontic \\
Have to & obligation & judgment/deontic \\
Need to & obligation & judgment/deontic \\
Should & opinion/advice & opinion/affective \\
Ought to & opinion/advice & opinion/affective \\
\hline
\end{tabular}

Meanwhile, in view of their justifications of glossary identifications, the authors draw a tentative analytical framework for stance glossary identification in this research (see Figure 1) for the present study. 


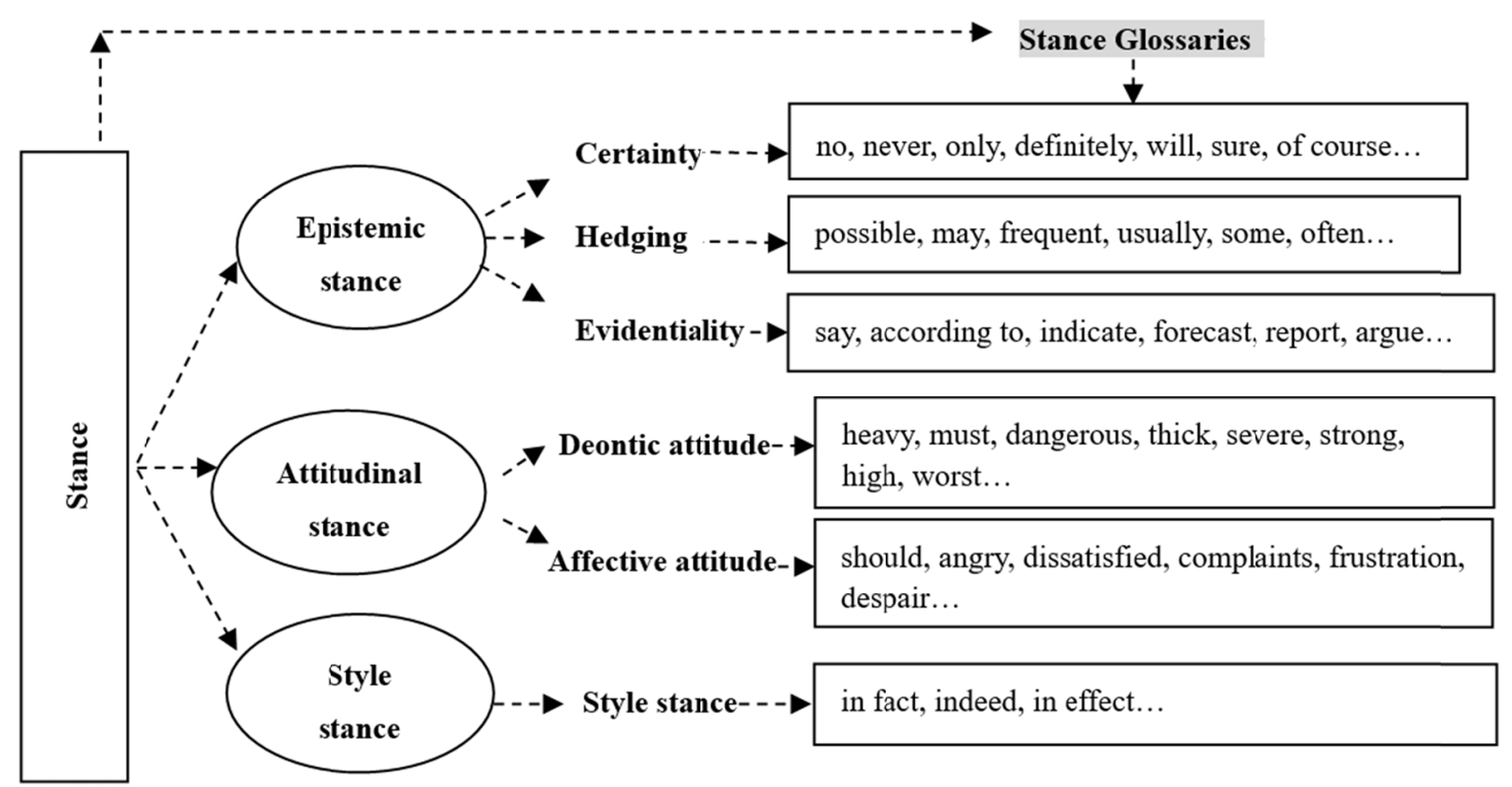

Figure 1. Tentative analytical framework of discourse stance and related glossaries in news discourses

Because this research is data-driven and focuses on 'tendencies', and 'probabilities', rather than single, isolated instances of "smog" in China, corresponding stance glossaries typical in the particular kinds of reports are identified and spelled out herewith for the convenience of later analysis and discussion.

\section{Data and Research Method}

\subsection{Data Collection}

The data to be analyzed in this research consists of existing authentic news reports, retrieved from the news database of LexisNexis Academic and Dow Jones Factiva, dated from December 2011 to April 2014. The authors applied multiple advanced computing searches, using the glossary extraction method by sourcing key words "smog" and "China". Regarding the topic of "smog" in China, the authors observed and chose 30 news reports respectively from American and Chinese authoritative news websites (see Table 2), such as the Chinese state news agency (Xinhua News Agency) and the China Daily newspaper in China, and Cable News Network $(\mathrm{CNN})$, the Time magazine, the Business Insider, the Wall Street Journal (WSJ), Yahoo, National Geographic magazine, International Business Times, Think Progress, US Today, Livescience, Extremetech, and Weather in the US. Only the body text of news report was taken into account, while the title and lead parts were omitted.

Table 2. Sources of English "smog" news in America and China

\begin{tabular}{llll}
\hline Country & Source of news & Number of pieces of news $($ Total = 60) & Period of collection \\
\hline America & Time & 10 & December, 2011-April, 2014 \\
$($ ASN) & CNN & 5 & December, 2011-April, 2014 \\
& Business Insider & 5 & December, 2011-April, 2014 \\
& WSJ & 2 & December, 2011-April, 2014 \\
& Others & 8 & December, 2011-April, 2014 \\
& Xinhua News Agency & 29 & December, 2011-April, 2014 \\
China & China Daily & 1 & December, 2011-April, 2014 \\
\hline
\end{tabular}

Note. $\mathrm{ASN}=$ American smog news, $\mathrm{CSN}=$ Chinese smog news.

The reason for collecting news written and posted during December 2011 to April 2014 is that weather conditions during that period deteriorated because heat provision system resumed in the winter across northern China. Accordingly, smog recurrently appeared in big cities so that news about it often hit headlines. Chinese-written news was extracted from the Xinhua News Agency and China Daily, while reports chosen from 
America were from more diverse sources. This selection was because Xinhua and China Daily, as China's most influential state media, provided sufficient English coverage of the smog problems, whereas it was difficult to collect adequate samples from only one or two news organizations in the US on the smog situation in China.

\subsection{Research Method}

The research methods employed in this study shift from qualitative analysis to quantitative analysis, with the hope of providing a comprehensive standpoint in decoding the cognitive stances in news reports on smog. The qualitative analysis on the data, together with the help of quantitative statistics, yields results with unbiased stances, verifying research findings on cross-cultural differences regarding English usage and the universal cognitive pattern of people in certain cultures via application of glossary extraction, which is also known as keyword extraction, terminology extraction, term extraction, or term recognition, as assistance for interpretation of the discoursal stance of news reports. In the present study, the extraction method of glossary classification is listed in Figure 2, following Beliga's (2014, pp. 1-2) suggestions on glossary assignments: glossary assignment and glossary extraction. In glossary assignment, glossaries are chosen from a controlled vocabulary of terms or predefined taxonomy, and documents are categorized into classes according to their content. Words that occurred in the document are analyzed in order to identify the most representative ones, usually exploring the source properties (i.e., frequency and length) (Zhang et al., 2008).

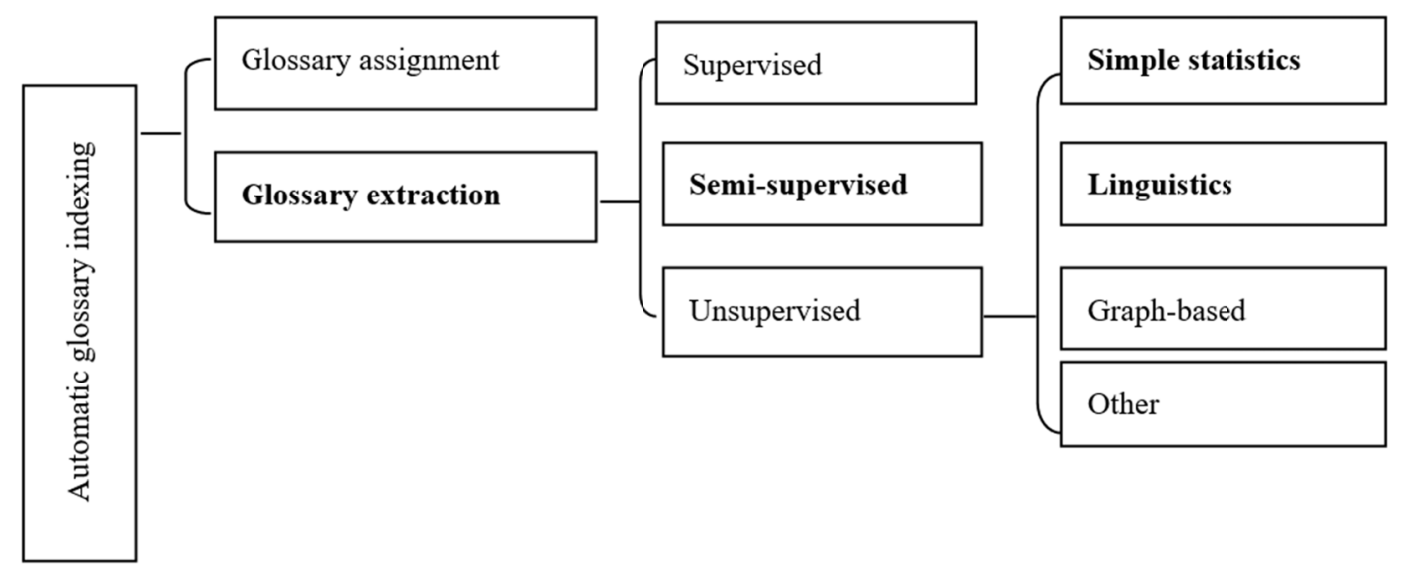

Figure 2. Classification of glossary extraction method (Beliga, 2014)

Although glossary extraction methods involve supervised, semi-supervised, and unsupervised measures, the present study utilizes the semi-supervised measure, i.e., manual annotations and simple statistics. According to Zhang et al. (2008), a simple statistics approach, i.e., term frequency-inverse document frequency (TFIDF) and a linguistic approach (part-of-speech tagging), is adopted to illustrate word frequency, word co-occurrences, and linguistic feature of the words in the smog discourses which are embedded in different cognitions. In the evaluation, there are three types of glossaries in manual assignment, which are suggested in Figure 1: epistemic glossaries, attitudinal glossaries, and style glossaries (for the convenience of identification, they are labeled as I, II, III). The previous two kinds have subsystems. Epistemic stance glossaries subsume certainty $\left(\mathrm{I}^{1}\right)$, hedging $\left(\mathrm{I}^{2}\right)$, and evidentiality $\left(\mathrm{I}^{3}\right)$. Attitudinal stance glossaries subsume deontic attitude $\left(\mathrm{II}^{1}\right)$ and affective attitude $\left(\mathrm{II}^{2}\right)$, while style stance glossaries (III) as the annotations are subsumed within the glossary extraction (see Table 3) too.

Table 3. Labels for glossary extraction and manual assignment

\begin{tabular}{lll}
\hline content & Glossary assignment by humans & Total extracted words \\
\hline Stance glossaries & A (I, II, III) & X \\
Subsume glossaries for extraction & $\mathrm{B}\left(\mathrm{I}^{1}, \mathrm{I}^{2}, \mathrm{I}^{3}, \mathrm{II}^{1}, \mathrm{II}^{2}, \mathrm{III}\right)$ & $\mathrm{Y}$ \\
\hline
\end{tabular}

In the data, the possible glossaries representing the news writers' cognitive stances are more than 30 lexical choices, which might be too complicated to use graphs and training data to interpret the semantic network in discourses. Hence, based on the information in Table 3, the authors have conducted an evaluation according to 
the general measure formula used in the information retrieval evaluation, i.e. stance frequency $(S F)$ and subsume glossary percentage $(G P)$. In this situation, $A$ and $B$ denote the number of instances of glossaries, and $X$ and $Y$ refer to the total instances of occurrence.

$$
\begin{aligned}
& S F(\mathrm{CSN}, \mathrm{ASN})=\frac{\mathrm{A}(\mathrm{I}, \mathrm{II}, \mathrm{III})}{X} \\
& G P(\mathrm{CSN}, \mathrm{ASN})=\frac{B\left(\mathrm{I}^{1} \ldots . \mathrm{III}\right)}{Y}
\end{aligned}
$$

\section{Findings and Discussion}

\subsection{Stance Analysis of Chinese Smog News (CSN)}

Smog news in English, as a typical typology of widely read public weather reports, are heavily embedded with social and political impacts because it is closely related to a national economic and industrial development, which exerts influences on the human environment. The result is that English smog reports have ceased being non-neutral anymore but are cognitively loaded. In this section, the authors conduct a manual annotation on the glossaries which represent the news writers' discourse stance and cognition on smog in China (see Example 1).

Example 1 (February 24, 2014 from China Daily):

\section{Smog to loom large over two sessions}

Environmental issues, especiallyII ${ }^{1}$ the nation's problems with smog, $\underline{\text { willI }}^{1}$ be a majorII ${ }^{1}$ focus at the upcoming plenary sessions of the National People's Congress and the National Committee of the Chinese People's Political Consultative Conference, $\underline{\operatorname{according~tol}}^{3}$ officials who are busyII ${ }^{1}$ preparing legislative proposals for the annual meetings.

"I am working on economic and technical proposals that willI ${ }^{1}$ implement the cleanII ${ }^{1}$ burning of coal, which will help solve our smog problem,"saidI ${ }^{3}$ Cao Xianghong, a petrochemical expert at the Chinese Academy of Engineering.

The proposal by Cao, a member of the National Committee of the CPPCC, willI $^{1}$ suggest $^{3}$ that the task of burning coal fall more heavilyII ${ }^{1}$ on large enterprises instead of individual households in order for the government to betterII ${ }^{1}$ manage emissions.

Yao Tandong, who last year recited a poem to President Xi Jinping during the two sessions to poke fun at the $\underline{\mathbf{h e a v y I I}}{ }^{1}$ smog, $\underline{\mathbf{s a i d l}}{ }^{3}$ his proposals $\underline{\text { willI }}^{1}{ }_{\text {likely }}{ }^{2}$ be about pollution. Yao is the director of the Chinese Academy of Sciences' Institute of Tibetan Plateau Research and a member of the National Committee of the CPPCC.

Chai Fahe, vice-president of the Chinese Research Academy of Environmental Sciences, $\underline{\text { saidI }}^{3}$ airborne pollution willI ${ }^{1}$ be a $\underline{\text { hotIII }}^{1}$ issue at the two sessions for years to come. Chai addedI ${ }^{3}$ that several legislators have inquired into the $\underline{\text { latestII }}^{1}$ draft of the nation's Atmospheric Pollution Prevention Act that willI ${ }^{1}$ be submitted to the NPC at the two sessions.

"The revision of the act $\underline{\text { willI }}^{1}$ be a major II $^{1}$ discussion point at this year's two sessions," he $\underline{\text { saidl }}^{3}$. "The legislators' proposals willI ${ }^{1}$ $\underline{\text { mainly }} I^{2}$ focus on three aspects: to accelerate the revision process, to clarify the responsibilities of local governments and polluters, which include strengthening penalties, and to enhance the management on mobile pollution sources."

Chai $\underline{\text { saidI }}^{3}$ many legislators $\underline{\text { willI }}^{1} \underline{\text { likely }}^{2}$ bring up the public's heightened II ${ }^{1}$ expectations of cleaner air as China releases its Airborne Pollution Prevention and Control Action Plan (2013-17) on the national level and as local governments create anti-pollution action plans. Leading up to the two sessions, combating smog was a top priority ${ }^{1}{ }^{1}$ at manyl $^{2}$ provincial legislative sessions in January and February. Last month, Beijing passed an airborne pollution prevention law that sets a pollution reduction target. The regulation provides a generalII ${ }^{1}$ framework for action that $\underline{\mathbf{w i l l}}^{1}$ be augmented by detailed directives to be published soon. It $\underline{\text { willI }}^{1}$ take effect on March 1 .

With many legislators focusing on battling smog, others said they $\underline{\text { willI }}^{1}$ focus on other environmental issues, such as water pollution and ecological security.

"I've already known enough deputies who are preparing to bring up smog in their proposals," said I ${ }^{3}$ Gao Jixi, director of the ecology institute of the Chinese Research Academy of Environmental Sciences.

He $\underline{\text { saidI }}^{3}$ he is working on a proposal about how to manage nature reserves to build an ecological network.

Gao, who is also a member of the CPPCC, $\underline{\mathbf{s a i d}}^{3}$ he $\underline{\mathbf{m a y}} \mathrm{I}^{2}$ also raise another proposal that tackles environmental problems during the process of urbanization.

ThickII ${ }^{1}$ smog has enveloped northern China for the past five days, affectingII ${ }^{1}$ more than one million square kilometers of the country.

Note. I: epistemic stance glossary, $\mathrm{I}^{1}$ : certainty, $\mathrm{I}^{2}$ : hedging, $\mathrm{I}^{3}$ : evidentiality, II: attitudinal stance glossary, $\mathrm{II}^{1}$ : deontic attitude, $\mathrm{II}^{2}$ affective attitude, III: style stance glossary.

In Example 1, there are forty-three stance glossaries in total in this selected sample, following the identification pattern in Figure 2. Among them, the main epistemic stance glossaries $I$ are:

(1a) Yao Tandong, who last year recited a poem to President Xi Jinping during the two sessions to poke fun at the heavy smog, $\underline{\text { saidl }}^{3}$ his proposals will $\mathrm{I}^{1} \underline{\text { likely }}^{2}$ be about pollution.

(1b) $\underline{\text { According to }}^{3}$ officials who are busy preparing legislative proposals for the annual meetings. 
(1c) The proposal by Cao, a member of the National Committee of the CPPCC, will $\mathrm{I}^{1} \underline{\text { suggest }}^{3}$ that the task of burning coal falls more heavily on large enterprises.

(1d) Chai added $I^{3}$ that several legislators have inquired into the latest draft of the nation's Atmospheric Pollution Prevention Act that will $\mathrm{I}^{1}$ be submitted to the NPC at the two sessions.

(1e) The legislators' proposals will $\mathrm{I}^{1}$ mainly $\mathrm{I}^{2}$ focus on three aspects.

Words such as "say", "according to", "add", "suggest" are often employed by news reporters to clarify source or quote reliable sources. "Will" is frequently applied to indicate the high possibility and certainty, while words such as "likely" and "may" are often used as hedges to represent uncertainty.

In excerpt (1a), the reporter quotes a representative who will hopefully put forward his own proposals about tackling pollution in the upcoming sessions. This proposal echoes the title "Smog to Loom Large over Two Sessions", and points out how this topic will catch attention. Additionally, "likely" here indicates uncertainty, conveying the reporter's discreetness and indirectly bespeaking the news reports' truth value.

In excerpt (1b), the reporter applies reliable source "officials" who are closely associated with two sessions to elicit the focus of the report. This quotation highlights the reliability of the news reports, and roots out any groundless personal deduction.

Excerpt (1c) goes deeper as the reporter not only confirms the existence of the concerned member's proposal, but also showcases the main content of the proposal—namely, that large enterprises shall bear more responsibilities than individuals for coal pollution. This quotation touches upon intentions as well as specific measures for amelioration of the smog problems in China.

In excerpt (1d), "add" plays the same role as "say". Its usage serves the same purpose of quoting a reliable source.

Excerpt (1e) again proves the accuracy of news reporting. The omission of "mainly" may incur misunderstanding that only the three aspects will be covered in all the proposals.

Next, attitudinal stance glossaries $\boldsymbol{I I}$ used in Example 1 indicate personal assessment or reveal emotional feelings.

(1f) Environmental issues, especially the nation's problems with smog, will be a major ${ }^{1}{ }^{1}$ focus at the upcoming plenary sessions.

(1g) According to officials who are busyII ${ }^{1}$ preparing legislative proposals for the annual meetings.

(1h) The task of burning coal falls more heavily ${ }^{1}{ }^{1}$ on large enterprises instead of individual households.

(1i) In order for the government to $\underline{\text { betterII }}^{1}$ manage emissions.

(1j) Yao Tandong, who last year recited a poem to President Xi Jinping during the two sessions to poke fun at the heavyII ${ }^{1}$ smog.

(1k) Airborne pollution will be a $\underline{\text { hotII }}^{1}$ issue at the two sessions for years to come.

(11) Leading up to the two sessions, combating smog was a top priority $\mathrm{II}^{1}$ at many provincial legislative sessions in January and February.

(1m) The regulation provides a generalII ${ }^{1}$ framework for action that will be augmented by detailed directives to be published soon.

(1n) ThickII $^{1}$ smog has enveloped northern China for the past five days.

(10) AffectingII ${ }^{1}$ more than one million square kilometers of the country.

In excerpts (1f) (1g) (1i) (1j) (1k) (1m) (1n), adjectives are employed to make judgments, whether they are from the reporters themselves or from quoted sources. "Thick" and "heavy" are frequently adopted as adjectives to modify the noun "smog", which not only informs readers of the severity, but also conveys the strong concerns of the reporters and passes these concerns to readers so as to direct their attention to the serious smog conditions.

Excerpts (1h) (11) (1o) demonstrate that adverbs, nouns, and verbs can also function as channels in making judgment. "More heavily" indicates the speaker's evaluation that, to a larger extent, enterprises rather than individuals should be responsible for lessening coal burning. "Top priority" indicates the urgency of tackling the environmental issues as many provincial sessions put it at the top of the agenda. "Affecting" itself, though normally a neutral word, here is more packed with negativity, implying that the smog hovering over in the sky will bring unfavorable consequences to the populace. 
To summarize, albeit epistemic and attitudinal glossaries are often seen, style stance glossaries are not typically applied, which structures the main CSN cognitive linguistic frame.

\subsection{Stance Analysis of American Smog News (ASN)}

Using the same analytical pattern that the authors employed in section 4.1, the authors selected an American sample to conduct the same discourse study, focusing on stance glossaries.

\section{Example 2 (February 21, 2014 from CNN):}

\section{Beiiing Issues Rare Pollution Alert As Smog Intensifies}

Beijing officials raised the city's air pollution alert on Friday to orange - the second-highest level of urgency —as smog levels have been projected to stay hazardous II $^{1}$ for locals through the weekend.

While the air quality has not been as hazardous ${ }^{1}{ }^{1}$ as in January, when city officials recorded extremely poisonous ${ }^{1}{ }^{1}$ air pollution, the $\underline{\text { rare II }}{ }^{1}$ orange alert prompts bans on barbeques, fireworks, and demolition works, the Associated Press reports ${ }^{3}$. Only the most $\underline{\text { severe }}^{1}$ red alert would put emergency plans into effect to pull half the city's cars off the road.

Both the elderly and children have been advised to stay indoors as current air quality levels are nine times the recommended levels set by the World Health Organization (WHO).

Beijing is one of the least hospitableII ${ }^{1}$ cities worldwide for its levels of air pollution, which often $I^{2}$ exceed limits set by the WHO. The

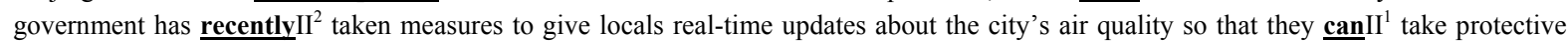
health measures.

(164 words)

Note. I: epistemic stance glossary, $\mathrm{I}^{1}$ : certainty, $\mathrm{I}^{2}$ : hedging, $\mathrm{I}^{3}$ : evidentiality, II: attitudinal stance glossary, $\mathrm{II}^{1}$ : deontic attitude, $\mathrm{II}^{2}$ affective attitude, III: style stance glossary.

In Example 2, ten stance glossaries are identified. Among them, only one epistemic stance glossary (evidentiality) is found while the rest all belong to deontic attitude. There is no trace of style stance glossaries. Examples are:

(2a) As smog levels have been projected to stay hazardousII ${ }^{1}$ for locals through the weekend.

(2b) When city officials recorded extremely poisonous $I^{1}$ air pollution.

(2c) The $\underline{\text { rareII }}^{1}$ orange alert prompts bans on barbeques, fireworks, and demolition works.

(2d) The Associated Press $\underline{\text { reports }}^{3}$.

(2e) Only the most severeII ${ }^{1}$ red alert would put emergency plans into effect...

(2f) Beijing is one of the least hospitable $I^{1}{ }^{1}$ cities worldwide for its levels of air pollution...

$(2 \mathrm{~g})$... the city's air quality so that they $\underline{\mathbf{c a n}}^{1}{ }^{1}$ take protective health measures.

In excerpts (2a), (2b) and (2e), the reporter underlines the high severity of smog and assesses the rise of smog condition to dangerous levels instead of using such words as "thick", "heavy" and "severe" smog as seen in CSN. In excerpt (2c) and (2f), both "rare" and "can" are categorized into attitudinal glossary because it is the reporter making the judgment that orange alerts are rarely issued in China and "Beijing" should be responsible for taking protective measures. Such a word used indirectly reflects the intensification of smog and can easily arouse the concern of readers.

Excerpt (2d) shows that "report" is another word indicating evidentiality. In Example 2, the reporter quotes another press statement to trace the fact that the foreign press doesn't enjoy the same opportunities as Chinese-based reporters due to language or national differences.

In excerpt (2e), the reporter uses the negative expression "least hospitable" when judging Beijing, which bears strong personal connotations.

To further elaborate on the discourse stances applied in media in both China and the US on smog in China, the authors analyze additional examples of glossary communicating certainty $\left(\mathrm{I}^{1}\right)$ and affective attitude $\left(\mathrm{II}^{2}\right)$, which are not covered in Examples 1 and 2.

Here are some more CSN examples.

Example 3 (selected from Xinhua, 27 February, 2014)

Smog has been a major concernII ${ }^{2}$ for the Chinese public since early 2013. Frequent bouts of smog have led to a reduction of industrial activities, raised pollution alerts and forced authorities to impose bans on vehicle use.

Example 4 (selected from Xinhua, 27 March, 2014) 
It is undeniables $^{1}$ that many deep-seated problems in economic and social development remain around China, and it will be impossiblel ${ }^{1}$ to resolve them completely in a short period of time, said Wang Zhongwu, a sociology professor at Shandong University. But "a good start is half way to success." said Wang.

The followings are ASN examples.

Example 5 (selected from International Business Times, 22 February, 2014)

Beijing's tiered air pollution alert system was introduced in October. But despite periods of heavy and dangerous smog, the plan's stricter measures have neveri ${ }^{1}$ been implemented.

Example 6 (selected from CNN, 16 January, 2013)

China mustII ${ }^{1}$ come clean on pollution.

Example 7 (selected from Time, 13 October, 2013)

The silver lining in the smog is that China's government is beginning to get serious about cleaning the air, thanks in no small part to popular angerII ${ }^{2}$ from average Chinese.

Affective attitude word "concern" in Example 3 indicates the fact that smog has raised anxiety among the public, while in Example 4, "undeniable" and "impossible" highlight the daunting smog-easing task, alluding to an enormous challenge that the government is already confronting. In Example 5 "never" conveys the absolute affirmation of editors in falling air quality, and in the government's failure to further respond. Modal verbs, such as "must" in Example 6, are adopted when editors are eager to cite solutions. Example 7 stresses wide-spread "anger" toward the government for its poor execution, mirroring the categorically negative attitude toward the Chinese government, insinuating that sloppy efforts were made with regard to environment-protection.

\subsection{Comparison Between CSN and ASN}

\subsubsection{Comparison of the Three Divisions of Stance Glossaries}

The previous analyses underline the different cognitive operations by media writers in selecting the most relevant linguistic choices for glossary assignments. The three varieties of stance glossaries are identified and counted from ASN and CSN reports respectively according to the authors' annotations. In Table 4, the statistical results of the two groups are listed for comparison. The data indicates that within the 30 selected reports, the total occurrences of epistemic stance glossaries are 481 in CSN, and 574 in ASN, among which both CSN and ASN writers prefer using epistemic stance glossaries to present the facts of smog in China, namely 288 and 264 respectively, and fewer relying on style stance glossaries, such as conjunctions like "in fact" and "generally speaking" in news reports. The big diversity is that ASN writers applied more attitudinal stance glossaries (307) than CSN writers did (196), indicating that ASN writers used more evaluative, personal, and individual opinion-related modal words like "must" for judgment and "should" for personal opinion in news reports.

Table 4. $S F$ taken by the three divisions of stance glossaries in smog news

\begin{tabular}{lllll}
\hline Category & Total & Epistemic & Attitudinal & Style \\
\hline CSN & 481 & $\mathbf{2 8 8}(\mathbf{6 0 \%})$ & $196(39.7 \%)$ & $2(0.4 \%)$ \\
ASN & 574 & $264(46 \%)$ & $\mathbf{3 0 7}(\mathbf{5 3 . 5 \% )}$ & $3(0.5 \%)$ \\
\hline
\end{tabular}

The results further display the discrepancies and similarities between the two groups regarding stance frequency $(S F)$ used in the news discourses. Both CSN and ASN writers use numerous epistemic stance markers that make up the largest portion regarding English lexical devices, $60 \%$ and $46 \%$ respectively, followed by attitudinal stance glossaries (39.7\% for CSN and $53.5 \%$ for ASN). This linguistic feature is not peculiar to "smog" news, because the differences of applying stance markers in discourses are not significant. As mentioned, one of the communicative purposes of news is to channel what happens to readers, which calls for quotations from as many reliable sources as possible to show the neutrality of the reporters. In epistemic stance samples, "will", as an epistemic stance marker showing certainty, is frequently used in the Chinese data samples (36 occurrences), whereas the American data sample contains only a few (14 occurrences). Nevertheless, one slight difference lies in the percentage variation of attitudinal stance glossaries, which, when compared to epistemic stance items, appear more often in ASN than those in CSN, demonstrating that public, social opinions, and personal evaluations are more widely applied in ASN than those in CSN. The authors noted that style stance glossaries are rarely used because it is generally assumed that weather information should be realistic and objective. 


\subsubsection{Comparison of Subdivisions of Stance Glossaries}

According to the authors' annotation, although the differences are not broadly evident, it is still necessary to break down the glossary percentages to explore the in-depth linguistic phenomenon regarding the linguistic subdivisions accounted for, as displayed in Table 5. This breakdown helps to identify the cognitive lexical usages in CSN and ASN discourses. The GP subdivisions provide linguistic evidence for the cognitive construction from a lexical perspective.

Table 5. GP subdivisions of stance glossaries in CSN and ASN discourses

\begin{tabular}{|c|c|c|c|c|c|c|c|c|c|c|c|c|c|}
\hline $\mathrm{CSN}$ & Epist & & & Attitud & & Style & ASN & Episten & & & Attitud & & Style \\
\hline & $\mathrm{c}$ & $\mathrm{h}$ & $\mathrm{e}$ & d & $\mathrm{a}$ & 1 & & $\mathrm{c}$ & $\mathrm{h}$ & $\mathrm{e}$ & $\mathrm{d}$ & $\mathrm{a}$ & 1 \\
\hline No. & 47 & 20 & 206 & 163 & 41 & 4 & No & 64 & 27 & 133 & 269 & 78 & 3 \\
\hline GP & $9.8 \%$ & $4.2 \%$ & $42.8 \%$ & $33.9 \%$ & $8.5 \%$ & $0.8 \%$ & GP & $11.1 \%$ & $4.7 \%$ & $23.2 \%$ & $46.9 \%$ & $13.6 \%$ & $0.5 \%$ \\
\hline Total & & & 481 & & & & Total & & & 574 & & & \\
\hline
\end{tabular}

Note. $\mathrm{c}=$ certainty, $\mathrm{h}=$ hedging, $\mathrm{e}=$ evidentiality, $\mathrm{d}=$ deontic, $\mathrm{a}=$ affective.

Regarding the three divisions of stance glossaries, noticeable disparities are detected. One disparity lies in the epistemic stance taking higher percentage in CSN, while the other rests on the percentage variation of attitudinal stance glossaries. Attitudinal stance glossaries, opposed to epistemic stance glossary, are highlighted more in ASN than they are in CSN. The statistical contrast also reveals differences in the subdivisions (certainty, hedging, evidentiality, deontic attitude glossaries, and affective attitude glossaries) on four fronts. In Table 5, semantically, CSN and ASN writers apply similar amounts of certainty and hedging glossaries in news discourses, showing the smog situation and existing environmental facts. As the authors mentioned, hedging glossaries used in news, though superficially contradicting to the conception of being definite, reflect careful wording by reporters in order not to convey incorrect or vague information. However, the result also illustrates the distribution of stance glossaries in which evidentiality and deontic stances markers are widely applied by both CSN and ASN writers with opposite preferences, showing that CSN writers generally prefer to apply official and institutional information in their smog reports, showing the sources of facts and evidence. On the other hand, ASN writers prefer to use evaluative lexical devices, following the public opinions and their own judgment. In the sample data, evidentiality glossaries application in CSN occupies $42.8 \%$, which is far more than the $23.2 \%$ of ASN, even though they may be found in all news discourses. In addition, deontic glossaries in ASN are $46.9 \%$, which outnumber those in CSN (33.9\%), indicating the judgmental, prescriptive, and evaluative viewpoints of reporters in relation to the topics. It is found that modal verbs, such as "must" and "should", are frequently used by ASN writers when they come to citing solutions to various problems that are indeed a serious environmental phenomenon calling for prompt actions, while CSN writers apply only a few in discourses to show their judgments and evaluation of the smog situation in China. Furthermore, owning to affective glossaries concerning the relationship between cognizing speakers/writers and their emotions (desire, anger, grief etc.) with respect to a given state of affairs, the average percentage $(G P)$ of affective glossaries in CSN (8.5\%) and ASN (13.6\%) suggests that weather news writers would prefer not to express their own emotions on smog issues.

\section{Discussion}

Although there are certain similarities regarding stance-taking in the press, the results suggest that the Chinese press is more official-oriented, while its American counterpart is more occupational-oriented. Lukin (2006, p. 79) suggests that news writers "should take into account not simply the obvious words or phrases that are topical in public debate, but the deeply unconscious patterns of choice involved in any use of language. In other words, it should be 'multi-dimensional', with respect to the many dimensions of linguistic 'choice' involved in the construction of a news story." The stance glossaries applied in both sets of samples demonstrate that the English applied by both the Americans and the Chinese is as much culturally co-determined as the glossaries of any other language, especially those expressing emotions, opinions, and positions.

First, ASN reporters are prone to use glossaries indicating certainty, and are eager to jump in and give commands to what should be done to mitigate smog by virtue of using high-valued lexical devices, which might impose reporters' own ideas.

Second, evidentiality glossaries in CSN are more common than those in ASN. Quoting sources intensify the reliability of news. However, regarding the selection of sources, CSN prefers citations from officials or relevant authoritative institutions (e.g., the Ministry of Environmental Protection), while ASN favors third-party sources, such as the Associated Press, the World Resource Institute, or even cynical citizens who are eager to vent their 
complaints. Such a difference demonstrates the fact that Chinese readers are more used to the rhetoric patterns of authority figures, and thus are more accustomed to quoting government officials. The Xinhua News Agency, the main source of CSN in this research, functions as the "throat" of the Chinese government, so more or less it serves the interests of the Chinese government. As a result, a government-oriented stance is pervasive throughout the news.

Third, the average percentage of deontic glossaries in ASN is relatively higher than that in CSN. While CSN covers neutral or positive words, ASN tends to employ more negative words ("poisonous", "least hospitable" etc.), constructing a negative image of China as a country neither livable nor hospitable. The American media, by intentionally selecting sources that transmit more pessimistic information, tries to frame China as a less clean country and its government as being lousy in execution of environmental protection. Compared with CSNs' government-oriented stance, ASNs are more press-oriented.

Finally, the average percentage of affective glossaries in ASN almost doubles that of CSN. What's more startling to find is that in CSN, affective glossaries are words like "public concern", "confident", etc., while ASN employs "discontent", "unbearable", "satirize", etc. Varied preferences for affective glossaries manifest that Chinese media are inclined to highlight the general public's positive responses to the remedies adopted by the Chinese government, while American media are skewed toward stressing the strong dissatisfactions of Chinese citizens with the thick smog, and with their complaints about the government's ineffective countermeasures. In media practice, Chinese and American media embrace two varied institutional stances: government stance and press stance. Different thinking patterns lead to such a varied stance taking as well as cognition framing. Thus, it is possible to work out the framing, as demonstrated in Figures 3 and 4.

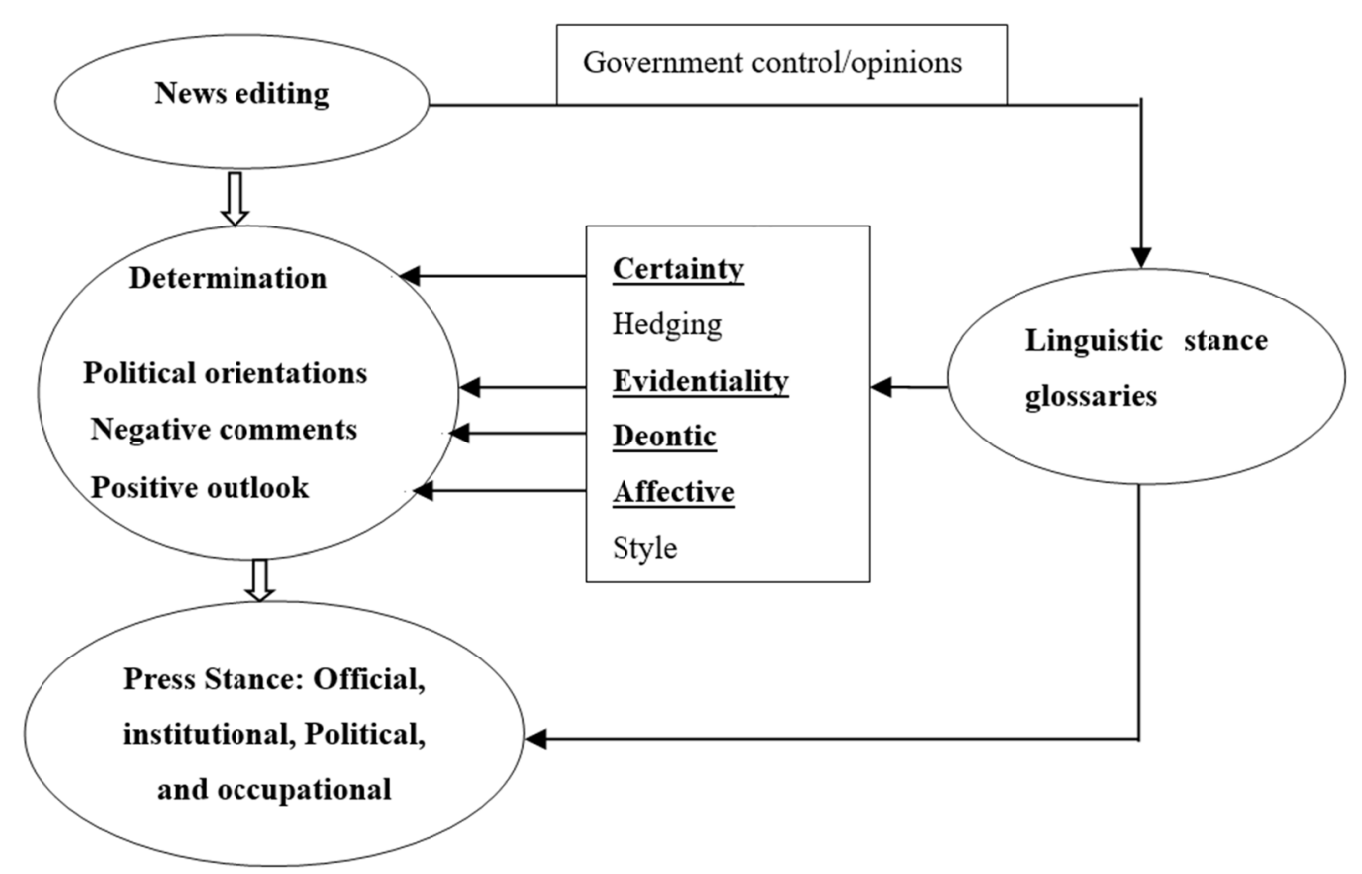

Figure 3. Cognitive frame reflected in CSN

According to Scheufele and Tewksbury (2006), there are at least five elements that may affect the framing of media reporting: social norms and values, organizational pressures and constraints, pressures of national interest, journalistic routines, and the ideological and political orientations of journalists. Xinhua News Agency, the source of CSN in this research, functions as a media institution of the Chinese government, i.e., Xinhua News Agency, serving the public and society, upholds the political directions and direct public opinion. The cognitive pattern projected from the news discourse on smog is rather political, official, and institutional - a government stance forming a pattern. Considering socialism in China, it is not surprising that the Chinese media are affiliated to the government, even as a tool of guiding public opinion. 


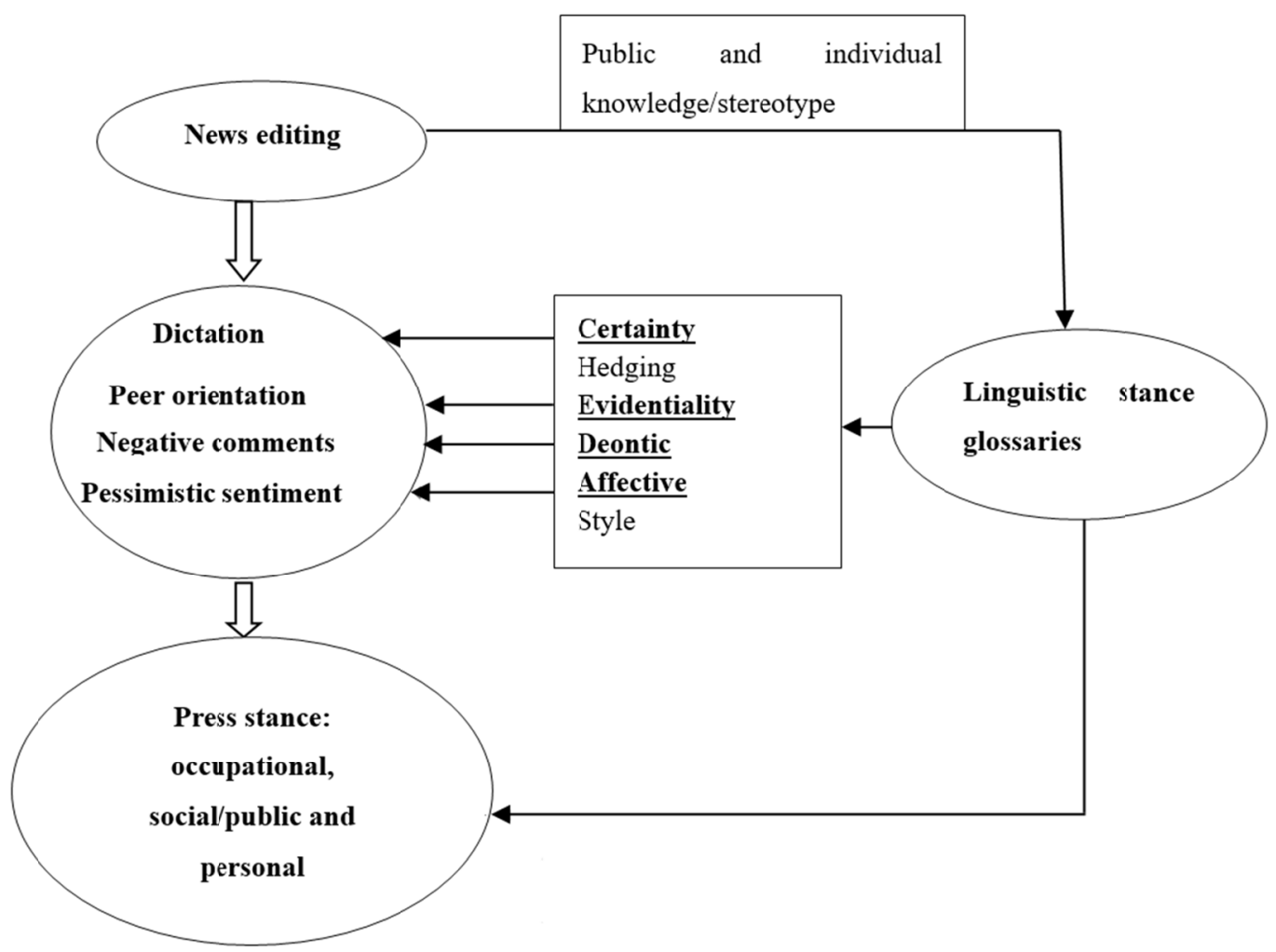

Figure 4. Cognitive frame reflected in ASN

As Gans (1979) argues, throughout the U.S. news judgment, the so-called eternal values are: ethnocentrism, altruistic national consciousness, reliable capitalism, individualism, modernism, social order, and national leadership, representing public opinion, such as those of institutions and individuals, dictations, sentiments, and comments on certain social issues. These timeless values find their way into news judgment. Also, due to the longstanding tendency to report on the negative aspects of China, preconceptions and prejudices have been implanted in the minds of Western people. The American media often draw a "picture" showing that the Chinese people habitually suffer from various problems, and often exhibit distrust or even suspicion toward reports originating from China. The American media displays a press stance driven by national interest and stereotypes, and the cognitive pattern projected from the news discourses on smog is more occupational, social, and personal.

Based on the above cross-cultural semantic analysis and discussion, the authors argue that smog itself cannot frame our cognitive world in terms of environmental and social issues, although the discourse and the English lexical choices in regard to smog reports can indeed do so. People's knowledge of the world emerges from language use, and vice versa, people's language use leads to acts of social interaction. Press writers construe their experience as it is obtained from institutions and society for the purpose of communicating that experience to their readers, which in turn has broader social-interactional purposes. Likewise, the readers tend to construal the lexical choices or discourse for even broader social purposes. Meanwhile, the present analysis on smog discourses reveals that people from different cultures tend to apply different linguistic-choices at the discoursal level, which would provide theoretical contributions regarding lexical items and social cultural factors in real-life discourse (Su, 2017; Stubbs, 1996). Moreover, the analysis of subdivisions of stance glossaries in smog news pattern can provide meaningful cognitive linguistic preferences and cognitive construction regarding lexical selections in CSN and ASN reports on China, which can offer implications for corpus linguistic designs and IR identifications in linguistics.

\section{Conclusion}

How events are reported is not an absolute reflection of the intrinsic importance of those events, but reveals processed coverage by a complex and artificial set of criteria. The news that has been thus selected is subjected to a transformational process before publication. This study echoes the argument by Enfield and Wierzbicka (2002, pp. 2-3): "While much work on emotion has assumed that English provides clear and non-ethnocentric terminology for the description of emotions in different ethno-linguistic spheres, some recent research has 
questioned this assumption. It has been shown that most linguistic categories (words, constructions) referring to emotions in natural languages embody complex and culture-specific configurations of ideas about how thoughts, feelings, and bodily processes may be normally (i.e., conventionally, in a given social realm) related. English-language 'technical' terminology is no exception, and it must thus be recognized that English-language descriptions of emotion are also 'folk descriptions', not culture-independent (descriptions)." Through this research, we have obtained the insights into cognition and language applications, projecting cognitive varieties in different cultures on certain social issues through the study of language, and vice versa.

\section{Acknowledgement}

This research has been supported by MOE Project of Humanities and Social Sciences, Ministry of Education, China on "Empirical Cross-cultural Studies on Social Discourses and Their Cognitive Frames" (No. 14JJD740011), by CSC visiting scholar research project (No. [2017]3059), and by MOE Center for Linguistics and Applied Linguistics, Guangdong University of Foreign Studies. We also appreciate Miss Alison Rae Mullins, an English expert who is teaching in Guangdong University of Foreign Studies, for her proofreading and language comments. We are also very grateful to the journal reviewers and editors for their comments for the revision of this paper. We are the persons who are solely responsible for all the remaining errors and shortcomings.

The authors declare that there is no conflict of interest regarding the publication of this paper.

\section{References}

Baratta, A. M. (2009). Revealing stance through passive voice. Journal of Pragmatics, 41(7), 1406-1421. https://doi.org/10.1016/j.pragma.2008.09.010

Bednarek, M., \& Caple, H. (2017). The discourse of news values: How news organizations create newsworthiness. New York: Oxford University Press. https://doi.org/10.1093/acprof:oso/9780190653934.001.0001

Beliga, S. (2014). Keyword extraction: Review of methods and approaches. Retrieved from https://pdfs.semanticscholar.org/bdbf/25f3dcf63d38cdb527a9ffca269fa0b8046b.pdf

Bell, A. (1991). The language of news media. Oxford: Blackwell.

Berman, R. A. (2005). Introduction: Developing discourse stance in different text types and languages. Journal of Pragmatics, 37(2), 105-124. https://doi.org/10.1016/S0378-2166(04)00189-4

Biber, D., \& Conrad, S. (2001). Quantitative corpus-based research: Much more than bean counting. TESOL Quarterly, 35(2), 331-336. https://doi.org/10.2307/3587653

Biber, D., Johansson, S., Leech, G., Conrad, S., Finegan, E., \& Quirk, R. (1999). Longman grammar of spoken and written English (Vol. 2). Cambridge MA: MIT Press.

Chandrasegaran, A., \& Kong, K. M. C. (2007). Stance-taking and stance-support in students' online forum discussion. Linguistics and Education, 17(4), 374-390. https://doi.org/10.1016/j.linged.2007.01.003

Damon, W., \& Phelps, E. (1989). Critical distinctions among three approaches to peer education. International Journal of Educational Research, 13(1), 9-19. https://doi.org/10.1016/0883-0355(89)90013-X

Enfield, N., \& Wierzbicka, A. (2002). The body in description of emotion. Pragmatics and Cognition, 10, 1-25. https://doi.org/10.1075/pc.10.12.02enf

Entman, R. M. (2010). Media framing biases and political power: Explaining slant in news of Campaign 2008. Journalism, 11(4), 389-408. https://doi.org/10.1177/1464884910367587

Fowler, R. (1991). Language in the news: Discourse and ideology in the press. London: Routledge.

Gales, T. (2011). Identifying interpersonal stance in threatening discourse: An appraisal analysis. Discourse Studies, 13(1), 27-46. https://doi.org/10.1177/1461445610387735

Gans, H. J. (1979). Deciding what's news: A study of CBS Evening News, NBC Nightly News, Newsweek, and Time. Chicago: Northwestern University Press.

Hunston, S., \& Thompson, G. (2000). Evaluation in text: Authorial stance and the construction of discourse. Oxford University Press.

Hyland, K. (2005). Stance and engagement: A model of interaction in academic discourse. Discourse Studies, 7(2), 173-192. https://doi.org/10.1177/1461445605050365 
Kärkkäinen, E. (2012). I thought it was very interesting: Conversational formats for taking a stance. Journal of Pragmatics, 44(15), 2194-2210. https://doi.org/10.1016/j.pragma.2012.09.005

Keck, C., \& Biber, D. (2004). Modal use in spoken and written university registers: A corpus-based study. In R. Facchinetti \& F. Palmer (Eds.), English modality in perspective: Genre analysis and contrastive studies (pp. 3-25). Frankfurt am Main: Peter Lang.

Lukin, A. (2006). What is media 'bias'? A case study of Al Jazeera's reporting of the Iraq War. Journal of Policing, Intelligence and Counter Terrorism, 1(1), 65-80. https://doi.org/10.1080/18335300.2006.9686879

Scheufele, D. A., \& Tewksbury, D. (2006). Framing, agenda setting, and priming: The evolution of three media effects models. Journal of Communication, 12(2), 9-20. https://doi.org/10.1111/j.0021-9916.2007.00326.x

Stubbs, M. (1996). Text and corpus analysis: Computer-assisted studies of language and culture. Oxford: Blackwell Publishers.

$\mathrm{Su}$, D. J. (2017). Significance as a lens: Understanding the Mandarin ba construction through discourse adjacent alternation. Journal of Pragmatics, 117, 204-230. https://doi.org/10.1016/j.pragma.2017.06.019

Ungerer, F., \& Schmid, H. (2001). An introduction to cognitive linguistics. Beijing: Foreign Language Teaching and Research Press.

Vannini, P., \& Mccright, A. (2007). Technologies of the sky: A socio-semiotic and critical analysis of televised weather discourse. Critical Discourse Studies, 4(1), 49-74. https://doi.org/10.1080/17405900601149475

Yang, W. H., Liang, Q. C., \& Zhen, K. Y. (2016). A discourse study of cognitive frame construction of 'China' in American economic news. English Language Teaching, 5(4), 7-24. https://doi.org/10.5430/elr.v5n4p7

Yang, W. H., Liu, X. F., \& Zhen, K. Y. (2018a). Current discourse space in Sino-American economic news on "The Belt and Road". Cross-Cultural Communication, 14(4), 25-42.

Yang, W. H., Zhao, J. P., \& Zhen, K. Y. (2018b). A schematic discoursal study of Chinese football commercial transfer news. Studies in Media and Communication, 6(2), 20-32. https://doi.org/10.11114/smc.v6i2.3575

Zhang, C., Wang, H., Liu, Y., Wu, D., Liao, Y., \& Wang, B. (2008). Automatic keyword extraction from documents using conditional random fields. Journal of Computational Information Systems, 4(3), 1169-1180. Retrieved from http://www.doc88.com/p-450274096192.html

\section{Copyrights}

Copyright for this article is retained by the author, with first publication rights granted to the journal.

This is an open-access article distributed under the terms and conditions of the Creative Commons Attribution license (http://creativecommons.org/licenses/by/4.0/). 